\title{
ON NESTICUS FROM MERIDIONAL SOUTH AMERICA (ARANEAE, NESTICIDAE)
}

\author{
Ricardo Ott ${ }^{1}$ \\ Arno Antonio Lise ${ }^{2}$
}

\begin{abstract}
Five new species of Nesticus Thorell, 1869 are described: N. potreiro, N. taim from southern Brazil; N. brignolii from southern Brazil, Argentina and Uruguay; N. calilegua from southern Brazil and Argentina and $N$. ramirezi, from Argentina. The male palpus and female epyginum of $N$. brasiliensis Brignoli, 1979 are illustrated. Described species were collected in epigean habitats, in shrubs, leaf-litter, under stones, bricks, tiles or debris.
\end{abstract}

KEYWORDS. Nesticus, Nesticidae, Araneae, Neotropical, taxonomy.

\section{INTRODUCTION}

Nesticus Thorell, 1869 is a very rich genus, with over 120 described species worldwide (PLATNICK, 2002). The majority are cavernicoles known from temperate areas (LEHTINEN \& SAaristo, 1980; GerTsCh, 1984). Few species are recorded from the Neotropical region, outstanding a dozen Central American and West Indies species (GERTCH, 1984; PlatNICK, 2002). Only four species are described from South America: N. citrinus Taczanowski, 1874 from French Guyana, N. unicolor Simon, 1894 from Venezuela, N. delfini Simon, 1904 from Chile and N. brasiliensis Brignoli, 1979 from Brazil (Brignoli, 1979; Platnick, 2002).

The genital characters of five new species, herein described, suggest that they should be assigned to Nesticus, despite some peculiarities. Males of these South American species have two processes at paracymbium, a single suprategular process and the course of spermatic ducts, in the suprategulum, configures a loop. Males of Holarctic species have, usually, a more complex paracymbium, an often fragmented suprategular process and sinuous spermatic ducts in the suprategulum (Lethinen \& SAARISTO, 1980; GerTsCH, 1984; Coyle \& McGarity, 1991). Females of the new species have typical Nesticus characters, with simple external genitalia and characteristic vulval glands (LETHINEN \& SAARISTO, 1980). The still unclear phylogenetic relationships of Nesticidae genera and the lack of revision papers, let the species assignment, to Nesticus, be tentative. Another

1. Museu de Ciências Naturais, Fundação Zoobotânica do Rio Grande do Sul, Rua Dr. Salvador França, 1427, 90690-000, Porto Alegre, RS, Brazil. (aracno@fzb.org.br)

2. Laboratório de Aracnologia, Pontifícia Universidade Católica do Rio Grande do Sul, Av. Ipiranga, 6681, Prédio 12C, Sala 244, 90619-900, Porto Alegre, RS, Brazil. (lisearno@pucrs.br) 
particularity of South American new species is that they were found on epigean habitats, such as shrubs near the ground, under stones, bricks, tiles, in litter or debris. According to Lethinen \& SAaristo (1980), Holarctic species are mainly restricted to caves or microcaverns.

The examined material is deposited in MCTP, Museu de Ciências e Tecnologia da Pontifícia Universidade Católica do Rio Grande do Sul, Porto Alegre (A. A. Lise); MCN, Museu de Ciências Naturais, Fundação Zoobotânica do Rio Grande do Sul, Porto Alegre (E. H. Buckup); MHNG, Museum D’Histoire Naturelle Genève (B. Hauser); MSNV, Museo Civico di Storia Naturale di Verona, Collezione Brignoli, Verona (R. Salmazo); USNM, National Museum of Natural History, Smithsonian Institution, Washington (D. G. Furth); IBSP, Intituto Butantan, São Paulo (A. D. Brescovit); MACN, Museo Argentino de Ciências Naturales Bernardino Rivadavia, Buenos Aires (C. L. Scioscia). All measurements are in millimeters. Female epigynes illustrations were made on removed ones; dorsal view drawings were made with the piece immersed in clove oil to clarify the internal structures.

\section{Nesticus brasiliensis Brignoli}

(Figs. 1-5, 38)

Nesticus brasiliensis Brignoli, 1979:915, figs. 5-8; $\sigma^{-1}$ holotype, $Q$ paratype, Nova Teutônia, Seara (300500 m), Santa Catarina, Brazil, VIII.1968, F. Plaumann (MHNG), examined; 9 paratype from same locality, XI.1958, F. Plaumann (MSNV), examined.

Diagnosis. Males of Nesticus brasiliensis can be recognized by the shape of the paracymbium processes, the ventral with a blunt end and the distal enlarged with a translucent area (figs. 2, 3); basal portion of conductor with a pointed process, distal portion with three conspicuous pointed process in ventral view (figs. 1,2); subtegulum conspicuous, visible in ventral and retrolateral view (figs. 2,3). Females are recognized by the shape of epigynum, with the posterior medium projection almost same width of spermatecae diameter; spermatecae are separated from each other less than their diameter (figs. 4, 5).

Description. See Brignoli (1979). A conspicuous thoracic fovea was omitted in the original description.

Material examined. BRAZIL, Rio Grande do Sul: Esmeralda, Q, 24.V.1975, A. A. Lise (MCN

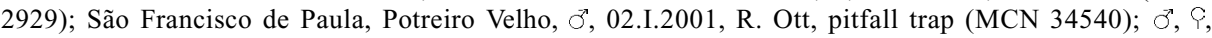
15-18.I.2002, L. A. Bertoncello, arboreal photoecletor (MCTP 13009).

Natural history. Specimens collected in Araucaria forest, inhabit, probably, ground and tree trunks. There are not detailed characteristics of habitat of the type-material.

Distribution. Known from the type locality (Nova Teutônia, Seara, Santa Catarina) and northeast of Rio Grande do Sul State, Brazil (fig. 38).

\section{Nesticus potreiro sp. nov.}

(Figs. 6-10, 30-34, 38)

Types. Holotype $\sigma^{-}$(MCTP 12541), Potreiro Velho (950 m), São Francisco de Paula, Rio Grande do Sul, Brazil, 26.X.1996, R. Ott \& A. A. Lise, under debris. Paratypes: 9

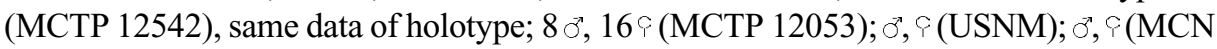


34334); đ̊ (, (MACN 10038), from same locality of holotype, 06.VII.1996, R. Ott. Etymology. The noun in apposition was taken from the type locality.

Diagnosis. Males of Nesticus potreiro differ from those of $N$. brasiliensis by shape of the paracymbial processes, the ventral finger-like and the distal with two terminal hooks, a dorsal pointed upward and a ventral, bifid, curved downward (figs. 9, 10, 30-32); basal portion of conductor rounded (figs. 8, 9), distal portion lack conspicuous prolateral tip like processes (figs. 8-10, 30, 32, 33); subtegulum conspicuous, visible in ventral and retrolateral views. Females are recognized by the shape of the epigynum, with posterior enlarged median projection (figs. 6, 7, 34), almost twice the spermatecae diameter; spermatecae separated from each other more than their diameter (figs. 6, 7).

Description. Male (holotype). Carapace yellowish with darkish spots on borders and a dark fork behind the eyes region, conspicuous fovea. Chelicerae and endites yellowish. Labium and sternum yellowish with some blackish points. Legs yellowish, with ventral blackish spots on the distal region of femora and tibiae. Abdomen grayish, with some discolored spots on dorsal region and on epigastric plates; wrinkled on the laterals.

Measurements. Total length 3.07. Carapace 1.48 long, 1.26 wide, 0.33 high. Clipeus 0.24 high. Chelicerae 0.64 long, 0.24 wide. Endites 0.48 long, 0.24 wide. Labium 0.14 long, 0.28 wide. Sternum 0.86 long, 0.79 wide. Eyes diameter and interdistances: AME 0.06, ALE 0.10 , PME 0.10, PLE 0.10, AME-AME 0.04, AME-ALE 0.02, PME-PME 0.10, PME-PLE 0.06 . MOQ 0.22 on both dorsal and frontal view, anterior wide 0.18 , posterior 0.26 . Abdomen 1.79 long, 1.26 wide, 1.33 high. Legs: I. femur 2.12 , patella 0.60 , tibia 1.86 , metatarsus 1.81 , tarsus 0.90 , total 7.29; II. 1.71, 0.57, 1.36, 1.33, 0.81, 5.78; III. 1.83, 0.50, 1.00, 1.02, 0.64, 4.99; IV. $2.00,0.60,1.74,1.69,0.83,6.86$. Palpus. $0.83,0.29,0.38,-, 0.60,2.10$.

Female paratype (MCTP 12542). Carapace yellowish, darkened on borders, two conspicuous darkened lines extended from the eyes region to the circular and low thoracic fovea. Chelicerae yellowish. Endites yellowish, slightly suffused of black. Labium and sternum as in male. Legs yellowish, suffused of black. Abdomen grayish olive, with some irregular dorsal discolored areas, also discolored on epigastric plates and pedicel.

Measurements. Total length 3.56. Carapace 1.60 long, 1.24 wide, 0.43 high. Clypeus 0.29 high. Chelicerae 0.71 long, 0.29 wide. Endites 0.38 long, 0.29 wide. Labium 0.14 long, 0.28 wide. Sternum 0.95 long, 0.81 wide. Eyes diameter and interdistances: AME 0.06, ALE 0.10, PME 0.10, PLE 0.10, AME-AME 0.04, AME-ALE 0.04, PME-PME 0.08, PME-PLE 0.06 . MOQ 0.24 long, dorsal view, 0.16 frontal view, 0.16 anterior wide, 0.28 posterior wide. Abdomen 2.31 long, 1.86 wide, 1.95 high. Legs: I. femur 1.93, patella 0.64, tibia 1.67, metatarsus 1.55 , tarsus 0.88 , total 6.67 ; II. 1.57, $0.57,1.19,1.10,0.71,5.14$; III. 1.26, $0.48,0.88$, $0.90,0.67,4.19 ;$ IV. 1.90, 0.62, 1.57, 1.38, 0.79, 6.26. Palpus. 0.64, 0.24, 0.36, -, 0.69, 1.93 .

Material examined. BRAZIL, Paraná: Almirante Tamandaré, Y, 06.IV.1984, E. C. Costa (MCN 12422); Y, 05.IV.1987, A. D. Brescovit (MCN 16722); Morretes, o', 9, 09-20.I.1995, PUCRS staff (MCTP 7200); Jaguariaíva (Parque Estadual do Cerrado), , VIII.1999, M. M. Braga (IBSP 24159). Rio Grande do Sul: São Francisco de Paula, Potreiro Velho (950m), 5 P, 06.VII.1996, R. Ott, under stones (MCTP 12543); 5 P, 06.VII.1996, R. Ott, inhabit inside abandoned herpetological pitfall traps (MCTP 12544); (Barragem Passo do Inferno), $9,26 . X I .2000$, E. Borsato, under stones (MCN 33256); (Barragem dos Bugres),, , 04.II.1999, L. A. Moura (MCN 31007); Canela,, , immature ${ }^{-2}$, 15.X.1965, A. A. Lise (MCN 392); ९, 10.II.1966, A. A. Lise (MCN 723); Y, 20.XII.1966, A. A. Lise (MCN 29321); ठ’, ९, 31.XII.1973, A. A. Lise (MCN 9874, 9872); Cambará do Sul, 3 9, 04.X.1975, A. A. Lise (MCN 3314); 2 ó, 09.I.1976, A. A. Lise (MCN 3349); (Itaimbezinho), Y, 03.X.1983, G. C. Lise (MCN 11807); 

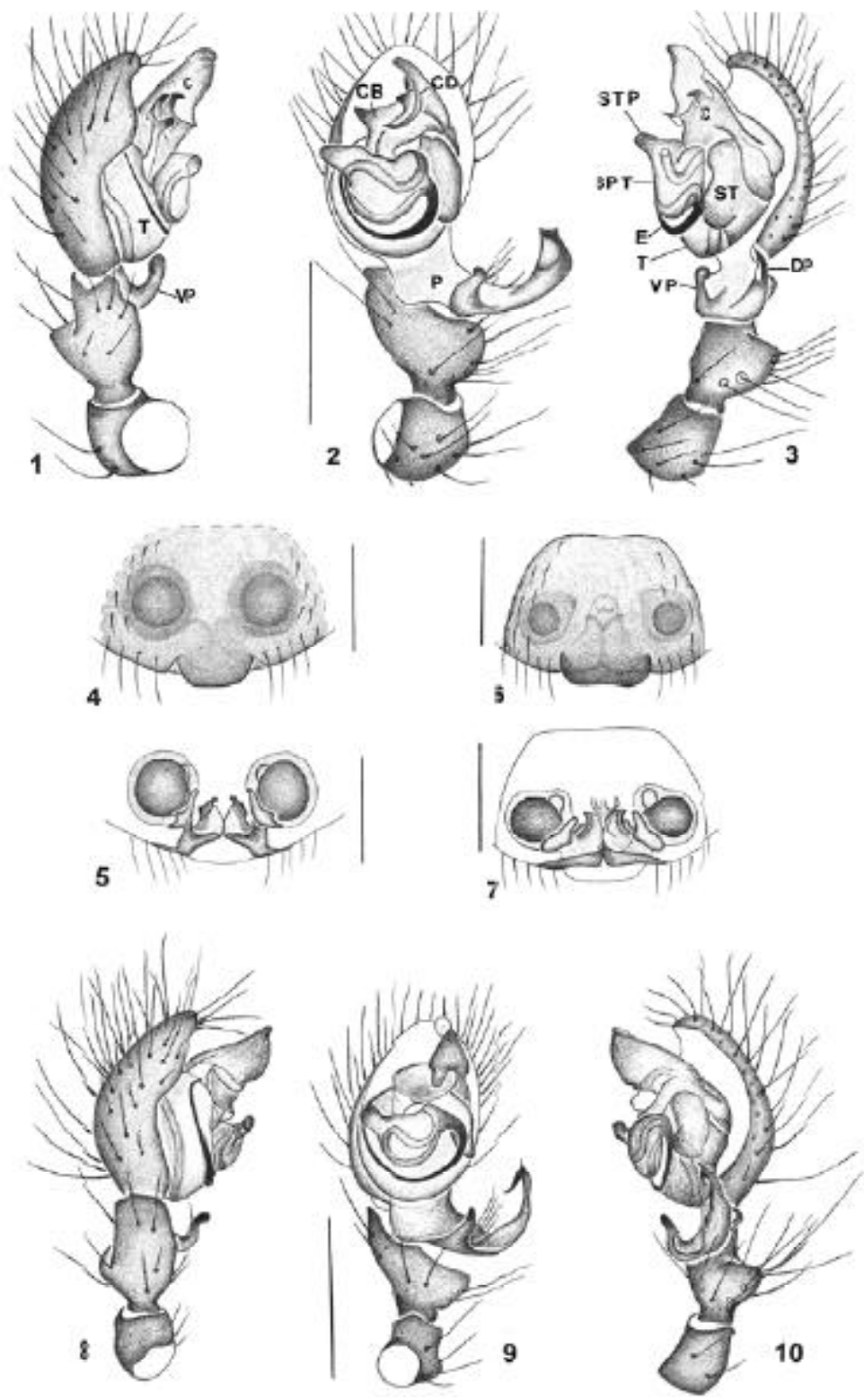

Figs. 1-10. Nesticus brasiliensis Brignoli, 1979: 1, male palpus, prolateral; 2, ventral; 3, retrolateral; 4, female epigynum, ventral; 5 , dorsal. Nesticus potreiro sp. nov.: 6 , female epigynum, ventral; 7 , dorsal; 8 , male palpus, prolateral; 9 , ventral; 10 , retrolateral. Scale bars: figs. $1-3,8-10,0.5 \mathrm{~mm}$; figs. 4-7, $0.25 \mathrm{~mm}(\mathrm{C}$, conductor; $\mathrm{CB}$, conductor basal portion; $\mathrm{CD}$, conductor distal portion; $\mathrm{DP}$, paracymbium distal process; E, embolus; SPT, suprategulum; ST, subtegulum; STP, suprategular process; T, tegulum; $\mathrm{VP}$, paracymbium ventral process).

Iheringia, Sér. Zool., Porto Alegre, 92(4):59-71, 30 de dezembro 2002 
(Passo do "S"), Y, 06.XI.1984, V. H. Travi (MCN 12655); Dom Pedro de Alcântara, Y, 18.IX.1975, A. A. Lise (MCN 3906); Gramado, Y, 11.XII.1976, C. J. Becker (MCN 4439); Caxias do Sul, Vila Oliva, 4Y, 14.IX.1976, C. J. Becker (MCN 4512); Passo Fundo, ơ, 25.VII,1977, A. A. Lise (MCN 6344); Osório, Vale do Maquiné, Q, 17.XI.1983, T. de Lema (MCN 11829); Machadinho, Y, 14.VII.1989, A. B. Bonaldo (MCN 18412).

Variation. Live specimens vary from dark brown to blackish; in alcohol the general coloration is mainly brown-olivaceous to yellowish, sometimes olivaceous. Males: total length 2.65-3.15; carapace length 1.35-1.50. Females: total length 2.80-4.10; carapace length $1.30-1.65$.

Natural history. This is a very common species at the highlands of Rio Grande do Sul (Araucaria plateau). Specimens were mainly observed and collected at open grasslands habitats near coppices and forests, generally under debris, stones, or in small holes on the ground (abandoned herpetological pitfall traps). Some specimens were found under construction remains, garbage and firewood, around human habitations, supposing a synantropic behavior. The spider was never observed inside of forests.

Distribution. Southern Brazil (fig. 38).

\section{Nesticus ramirezi sp. nov.}

(Figs. 11-16, 38)

Types. Holotype (MACN 10025), Parque Provincial Cruce Caballero, Misiones, Argentina, 27-29.X.1995, M. Ramírez. Paratype 9 (MACN 10026), Parque Nacional Iguazú, Misiones, Argentina, 11-16.XII.1999, M. Ramírez \& L. Lopardo.

Etymology. The specific name is a patronym honoring the Argentinean arachnologist Mártin Ramírez.

Diagnosis. Males of Nesticus ramirezi can be distinguished from those of $N$. brasiliensis and $N$. potreiro by the shape of paracymbium processes, the ventral Cshaped, the distal with three hooks (one median and two distal hooks) (figs. 11-13); broad conductor with a rounded process at basal portion and a median point at distal portion (figs. 11, 12); subtegulum conspicuous, visible in ventral and retrolateral views (figs. 12, 13). Females can be recognized by the epigynum, with a wide rounded posterior median projection (nose-like) and circular lateral depressions; spermatecae apart from each other about their diameter (figs. 14, 15).

Description. Male (holotype). Carapace tawny with laterals marbled with black; middle with a trident-like mark, extending behind the ocular area to the thoracic fovea; laterals marbled along entire carapace. Eyes circled with black. Thoracic fovea circular, shallow (fig. 16). Chelicerae, endites and labium yellow. Sternum yellow, shadowed of black; points of insertion of bristles yellow. First legs and third and fourth right legs, missing. Leg II yellow with blackish bands on apex of femur and patella and on the base and apex of tibia. Dorsum of abdomen whitish with some black spots; laterals and venter whitish with epigastric area shadowed of black; tegument with micro setae.

Measurements. Total length 1.70. Carapace 0.90 long, 0.74 wide, 0.30 high. Clypeus 0.12 high. Chelicerae 0.30 long, 0.16 wide. Endites 0.22 long, 0.18 wide. Labium 0.08 long, 0.18 wide. Sternum 0.54 long, 0.52 wide. Eyes diameter and interdistances: AME 0.04, ALE 0.10 , PME 0.10, PLE 0.10, AME-AME 0.02, AME-ALE 0.02, PME-PME 0.06, PME-PLE 0.02 . MOQ 0.14 long, frontal view, 0.16 dorsal view. Anterior wide 0.12 , posterior wide 0.22 . 

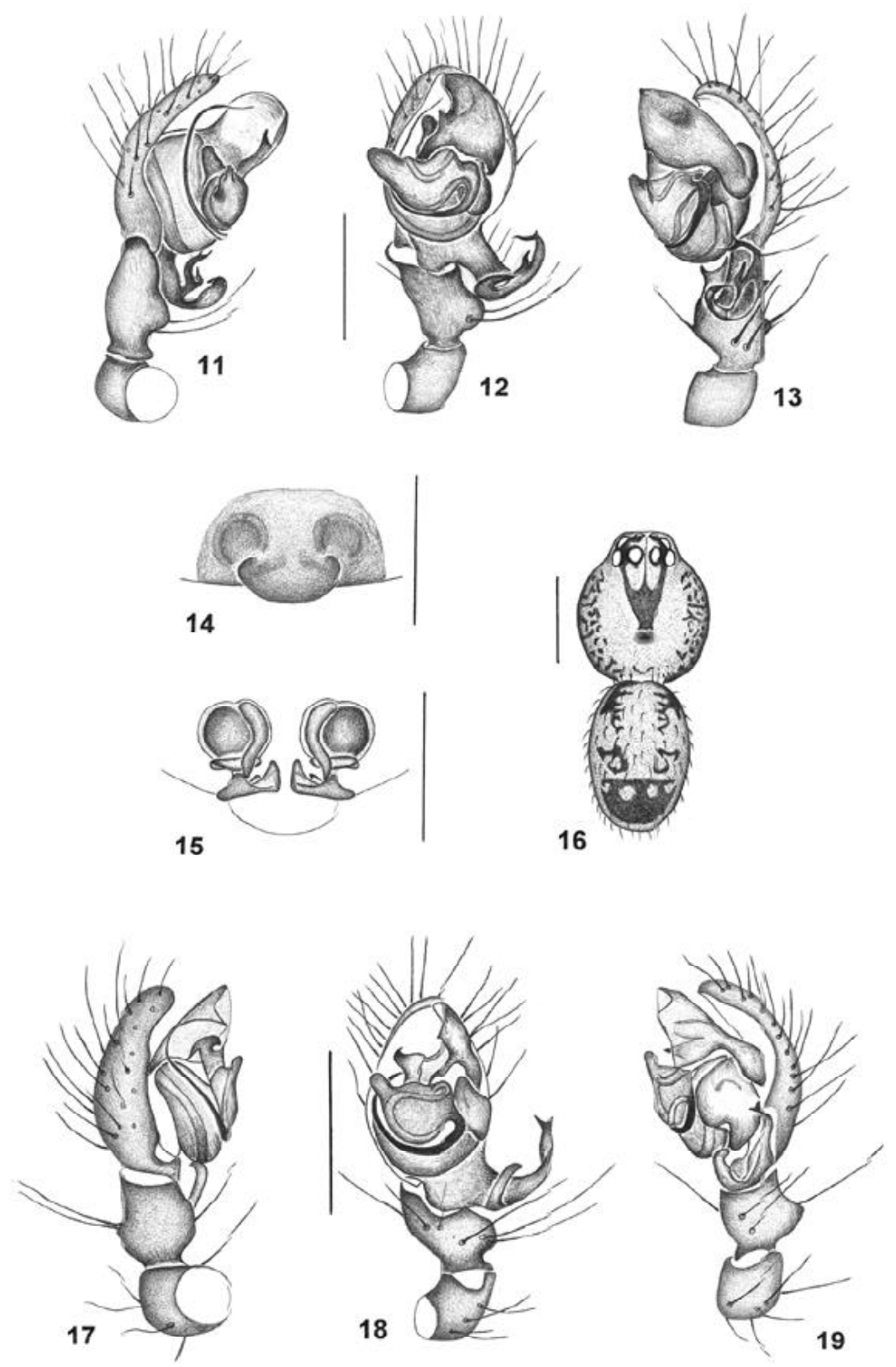

Figs. 11-19. Nesticus ramirezi sp. nov.: 11, male palpus, prolateral; 12, ventral; 13, retrolateral; 14, female epigynum, ventral; 15, dorsal; 16, male body, dorsal. Nesticus taim sp. nov.: 17, male palpus, prolateral; 18, ventral; 19, retrolateral. Scale bars: figs. $11-15,17-19,0.25 \mathrm{~mm}$; fig. $16,0.5 \mathrm{~mm}$.

Iheringia, Sér. Zool., Porto Alegre, 92(4):59-71, 30 de dezembro 2002 
Abdomen 0.80 long, 0.64 wide, 0.66 high. Legs: I. missing; II. femur 1.06, patella 0.32, tibia 0.90 , metatarsus 0.80 , tarsus 0.56 , total 3.64 ; III. $0.84,0.30,0.60,0.60,0.56,2.90$; IV. 1.40, 0.36, 1.16, 1.00, 0.60, 4.52. Palpus. 0.42, 0.14, 0.14, -, 040, 1.10 .

Female (paratype). Carapace almost elliptic, yellow greenish suffused of black; center with a trident-shaped black mark; smooth, with three large bristles on the center of the trident and two on eye region, one between ALE and PME and one backwards the AME; thoracic fovea circular. Clypeus with a median longitudinal cup-shaped black spot. Chelicerae yellow moderately suffused of black. Endites and labium tawny. Sternum yellow densely suffused of black. Legs: left IV detached, right I, III and IV missing, II detached; femur, patella and tibia yellow banded of black on the apical third; metatarsus and tarsus fulvous yellowish. Abdomen dark grayish with one white spot above the anal tubercle and another above the pedicel; the anterior third of median dorsal surface somewhat lighter; laterals with anterior elliptic gray spot each side, not well demarcated; venter dark grayish with two elliptic ligther spots on each side of epigynum.

Measurements. Total length 2.40. Carapace 1.06 long, 0.84 wide, 0.20 high. Clypeus 0.14 high. Chelicera 0.32 long, 0.18 wide. Endite 0.24 long, 0.18 wide. Labium 0.10 long, 0.18 wide. Sternum 0.56 long, 0.32 wide. Eyes diameter and interdistances: AME 0.06, ALE 0.10, PME 0.10, PLE 0.08, AME-AME 0.04, AME-ALE 0.02, PME-PME 0.06, PME-PLE 0.03. MOQ 0.16 long, frontal view, 0.20 dorsal view, 0.14 anterior wide, 0.24 posterior wide. Abdomen 1.40 long, 1.20 wide, 1.40 high. Legs: I. femur 1.40, patella 0.40, tibia 1.24, metatarsus 1.00, tarsus 0.66, total 4.70; II. 1.00, 0.34, 0.84, 0.74, 0.54, 3.46; III. 0.84, 0.32, 0.58, 0.54, 0.48, 2.76; IV. 1.26, 0.36, 1.12, 0.90, 0.60, 4.24. Palpus. 0.83, 0.14, 0.20, -, 0.42, 1.14 .

Natural history. According to labels, specimens are probably litter dwellers collected with Berlese funels.

Distribution. Known from Misiones, Argentina (fig. 38).

\section{Nesticus taim sp. nov.}

(Figs. 17-19, 38)

Type. Holotype (MCN 13587), Estação Ecológica do Taim, Rio Grande, Rio Grande do Sul, Brazil, 17.X.1985, E. H. Buckup.

Etymology. The noun in apposition was taken from the type locality.

Diagnosis. Males of Nesticus taim can be recognized by the shape of the male paracymbial processes, with the ventral slender and curved and the distal forked (figs. 18, 19); basal portion of conductor with a hook-like process (figs. 17, 18); subtegulum conspicuous, visible in ventral and retrolateral view (figs. 18, 19).

Female unknown.

Description. Male (holotype). Carapace yellowish with some slightly darkened points at middle and margins. Fovea small and circular. Eye region almost cephalic width. Chelicerae and endites yellowish. Labium yellowish, triangular. Sternum yellowish suffused of black, enlarged at posterior region. Legs yellowish, with ventral darkened areas on coxa and patella. Distal femur slightly suffused of black. Abdomen pale, with large dark grey spots on dorsum, two smaller spots on anterior region and a large u-shaped posterior spot. Venter with a large black anterior spot, extended from epigastric furrow to pedicel. Posterior region with large black ring surrounding spinnerets.

Measurements. Total length 1.37. Carapace 0.71 long, 0.60 wide, 0.26 high. Clypeus 
0.10 high. Chelicerae 0.31 long, 0.12 wide. Endites 0.14 long, 0.17 wide. Labium 0.06 long, 0.16 wide. Sternum 0.50 long, 0.40 wide. Eyes diameter and interdistances: AME 0.02, ALE 0.08, PME 0.08, PLE 0.08, AME-AME 0.02, AME-ALE contiguous, PME-PME 0.04, PMEPLE contiguous. MOQ 0.12 long, dorsal view, 0.10 frontal view, anterior wide 0.08 , posterior 0.16 . Abdomen 0.74 long, 0.52 wide, 0.55 high. Legs: I. femur 0.88 , patella 0.92 , tibia 0.79 , metatarsus 0.67 , tarsus 0.45 , total 3.71 ; II. $0.69,0.26,0.57,0.50,0.36,2.38$; III. 0.57, $0.24,0.40$, $0.38,0.33,1.92$; IV. $0.86,0.29,0.71,0.55,0.40,2.81$. Palpus. 0.36, 0.12, $0.17,-, 0.29,0.94$.

Natural history. Type collected at shrub vegetation (collector personal communication).

Distribution. Known only from Estação Ecológica do Taim, Rio Grande, Rio Grande do Sul, Brazil (fig. 38).

\section{Nesticus brignolii sp. nov.}

(Figs. 20-24, 35-38)

Types. Holotype (MCTP 12041), Guaíba, Rio Grande do Sul, Brazil, 14.VII.1995, A. A. Lise et al. Paratypes: 9 (MCTP 12042), same data of the holotype; (MCTP 12043), 22.IX.1995; 9 (MCTP 12046), 21.VIII.1994; 2 (MCTP 12045), 02.I.1995; 29 (MCTP 12049),

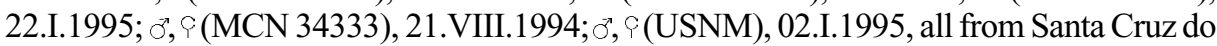
Sul, Rio Grande do Sul, Brazil, R. Ott.

Etymology. The specific name is a patronym in honor of the Italian arachnologist Paolo Marcelo Brignoli.

Diagnosis. Nesticus brignolii males can be recognized by the two hooked, paracymbial processes (figs. 21, 22, 35, 36), by the copulatory bulb, with tegulum larger than cymbium (fig. 21); basal portion of conductor rounded (fig. 20,21,37) and distal with a quadrangular process where embolus arrives (figs. 20,21,37); subtegulum very slender, hidden by tegulum, not visible in ventral view and inconspicuous in retrolateral view (figs. 21,22). Females can be recognized by the shape of epigynum, with coiled copulatory ducts and oval spermatecae (figs. 23, 24).

Description. Male (holotype). Carapace pale yellow darkened on margins, with a dark fork-like mark on dorsum; fovea inconspicuous. Chelicerae, endites and labium yellowish. Sternum yellowish suffused of darkened points. Legs pale yellow. Abdomen grayish, rounded, with some discolored spots on dorsal region, wrinkled on laterals and above the spinnerets.

Measurements. Total length 1.40. Carapace 0.74 long, 0.62 wide, 0.26 high. Clypeus 0.14 high. Chelicerae 0.33 long, 0.17 wide. Endites 0.21 long, 0.17 wide. Labium 0.06 long, 0.12 wide. Sternum 0.45 long, 0.43 wide. Eyes diameter and interdistances: AME 0.04, ALE 0.08 , PME 0.08, PLE 0.08, AME-AME 0.02, AME-ALE and PME-PLE contiguous, PMEPME 0.04. MOQ 0.12 long, on both, dorsal and frontal view. Abdomen 0.74 long, 0.50 wide, 0.48 high. Legs: I. femur 0.74 , patella 0.24 , tibia 0.62 , metatarsus 0.55 , tarsus 0.40 , total 2.55 ; II. $0.60,0.26,0.43,0.38,0.33,2.00$; III. $0.48,0.24,0.36,0.32,0.31,1.71$; IV. 0.74, 0.29 , $0.62,0.52,0.38,2.55$. Palpus. $0.33,0.12,0.12,-, 0.40,0.97$.

Female paratype (MCTP 12042). Carapace pale yellow, slightly darkened on margins and on the posterior end of the cephalic region. Chelicerae, endites and labium yellowish. Sternum yellowish, slightly spotted with black. Legs yellowish. Abdomen grayish, oval, with a wrinkled area above the spinnerets. 

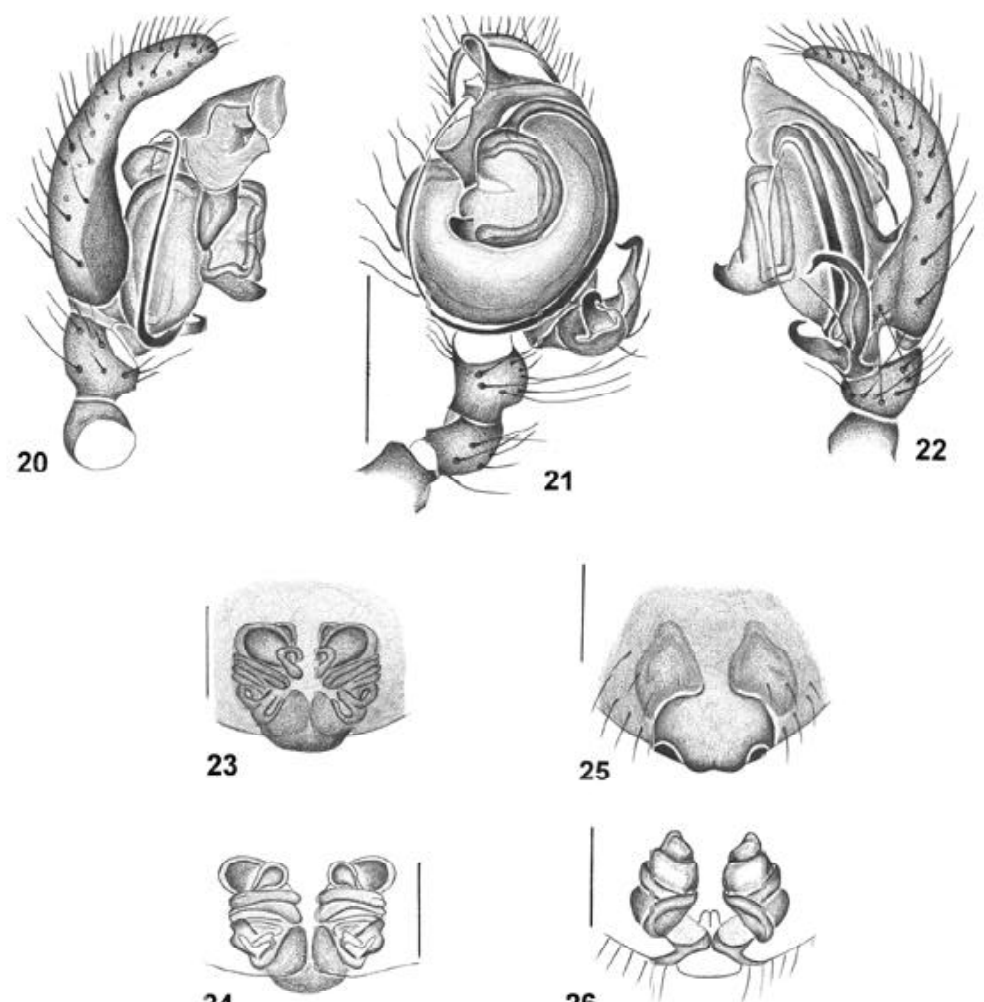

25

24
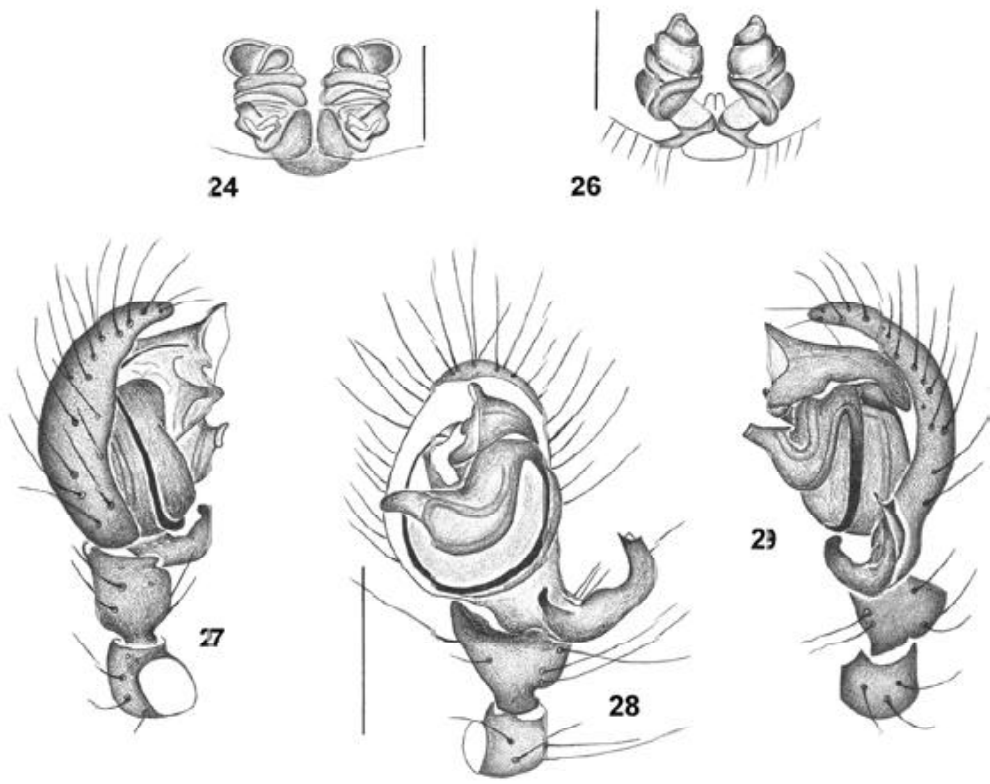

Figs. 20-29. Nesticus brignolii sp. nov.: 20, male palpus, prolateral; 21, ventral; 22, retrolateral; 23, female epigynum, ventral; 24 , dorsal. Nesticus calilegua sp. nov.: 25 , female epigynum, ventral; 26 , dorsal; 27, male palpus, prolateral; 28, ventral; 29, retrolateral. Scale bars: figs. $20-26,0.25 \mathrm{~mm}$; figs. $27-29,0.5 \mathrm{~mm}$. 
Measurements: Total length 3.56. Carapace 1.60 long, 1.24 wide, 0.43 high. Clypeus 0.29 high. Chelicerae 0.71 long, 0.29 wide. Endites 0.38 long, 0.29 wide. Labium 0.06 long, 0.16 wide. Sternum 0.95 long, 0.81 wide. Eyes diameter and interdistances: AME 0.04, ALE 0.06, PME 0.06, PLE 0.08, AME-AME 0.04, AME-ALE and PME-PLE contiguous, PMEPME 0.04. Abdomen 2.31 long, 1.86 wide, 1.95 high. Legs: I. femur 0.71 , patella 0.29 , tibia 0.52 , metatarsus 0.45 , tarsus 0.38 , total 2.35 ; II. $0.55,0.26,0.38,0.33,0.33,1.85$; III. $0.48,0.24$, $0.31,0.31,0.31,1.65 ;$ IV. $0.71,0.29,0.57,0.43,0.36,2.36$. Palpus. $0.26,0.14,0.14,-, 0.31,0.85$.

Material examined. BRAZIL, Rio Grande do Sul: São Francisco de Paula, ơ, 14.V.1993, A. Fonseca (MCTP 3475); Canela (Barragem dos Bugres), 4 9, 04.II.1999, A. B. Bonaldo, L. A. Moura (MCN 31004, 31105); Y, 02.II.1999, A. B. Bonaldo (MCN 31094); Y, 06.XI.1998, A. B. Bonaldo (MCN 31086); O', 13.XII.1999, A. B. Bonaldo (MCN 32090); Santa Cruz do Sul, 2 ó, 12.VIII.1995 (MCTP 12050); 2 O', 27.XI.1994 (MCTP 12051); O', 24.VII.1995 (MCTP 12052); Q, 02.I.1995 (MCTP 12045) all R. Ott; Guaíba, P, 03.X.1995, A. A. Lise et al. (MCTP 12044); Viamão, O’, 07.VII.1995, A. A. Lise et al. (MCTP 12048); Santa Maria (Morro do Elefante), Ó, ९, 1995, M. A. Somavilla (IBSP 7087). ARGENTINA, Misiones: (Parque Nacional Iguazú), P, 08-15.II.1995, M. Ramírez (MACN 10027); P, 14-16.XII.1999, M. Ramírez \& L. Lopardo (MACN 10028); O', 23 26.X.1995, M. Ramírez (MACN 10029); (Parque Provincial Uruguay, Refúgio Caa-Porà), C, 15.II.1995, M. Ramírez (MACN 10031). URUGUAY, Minas: Lavalleja (Cerro Arequita), P, 03.XII.1997, M. Ramírez \& L. Campagnucci (MACN 10030).

Variation. Total length: 1.40-1.70 males, $1.50-1.80$ females. Carapace: 0,73-0,75 males, 0.71-0.74 females.

Natural history. Specimens were collected in litter.

Distribution. Known from south Brazil (Rio Grande do Sul), Uruguay and north Argentina (fig. 38).

\section{Nesticus calilegua sp. nov.}

(Figs. 25-29, 38)

Types. Holotype $\sigma^{-1}$ MACN 10032), Parque Nacional Calilegua, Jujuy, Argentina, 23,24.IX.1995, M. Ramírez, P. Goloboff \& C. Szumik. Paratypes: $९$ (MACN 10033), same data of the holotype; $\sigma^{\prime}$, (MCTP 12054), Pampa de Achala, $15 \mathrm{~km} \mathrm{~W}$ el Condor, Cordoba, 31.VII.1999, M. Ramírez \& L. Lopardo; (MACN 10034), Alta Gracia, Cordoba, VII.1981, A. Zanetic; 9 (MACN 10035), Finca Jakulica, $25 \mathrm{~km}$ NW of Aguas Blancas, Salta, 1519.XI.1994, M. Ramírez \& P. Goloboff ; 2 (MACN 10036), Ojo de Agua, Ruta Nacional 9, Km 1640-1642, Salta, 22.IX.1995, M. Ramírez, P. Goloboff \& C. Szumik; 2 (MACN 10037), Mesada las Colmenas (1500m), Parque Nacional Calilegua, Jujuy, 8.VIII.1997, M. Ramírez \& L. Compagnucci, all from Argentina; ơ (MCN 27260), Morro do Baú, Ilhota, Santa Catarina, Brazil, 04.II.1996, A. B. Bonaldo.

Etymology. The noun in apposition was taken from type locality.

Diagnosis. Males of Nesticus calilegua can be recognized by the shape of the paracymbial processes, the ventral one curved, and the distal dorsally canaliculated, with three pointed ends (figs. 28, 29); conductor basal portion somewhat rectangular, distal portion with a rounded tip where embolus arrives (figs. 27, 28); subtegulum, not visible in ventral view (like in $N$. brignolii), visible in retrolateral (fig. 29). Females can be recognized by the shape of the epigynal posterior median projection, indented on each side and by the reniform spermatecae with coiled ducts (figs. 25, 26). 

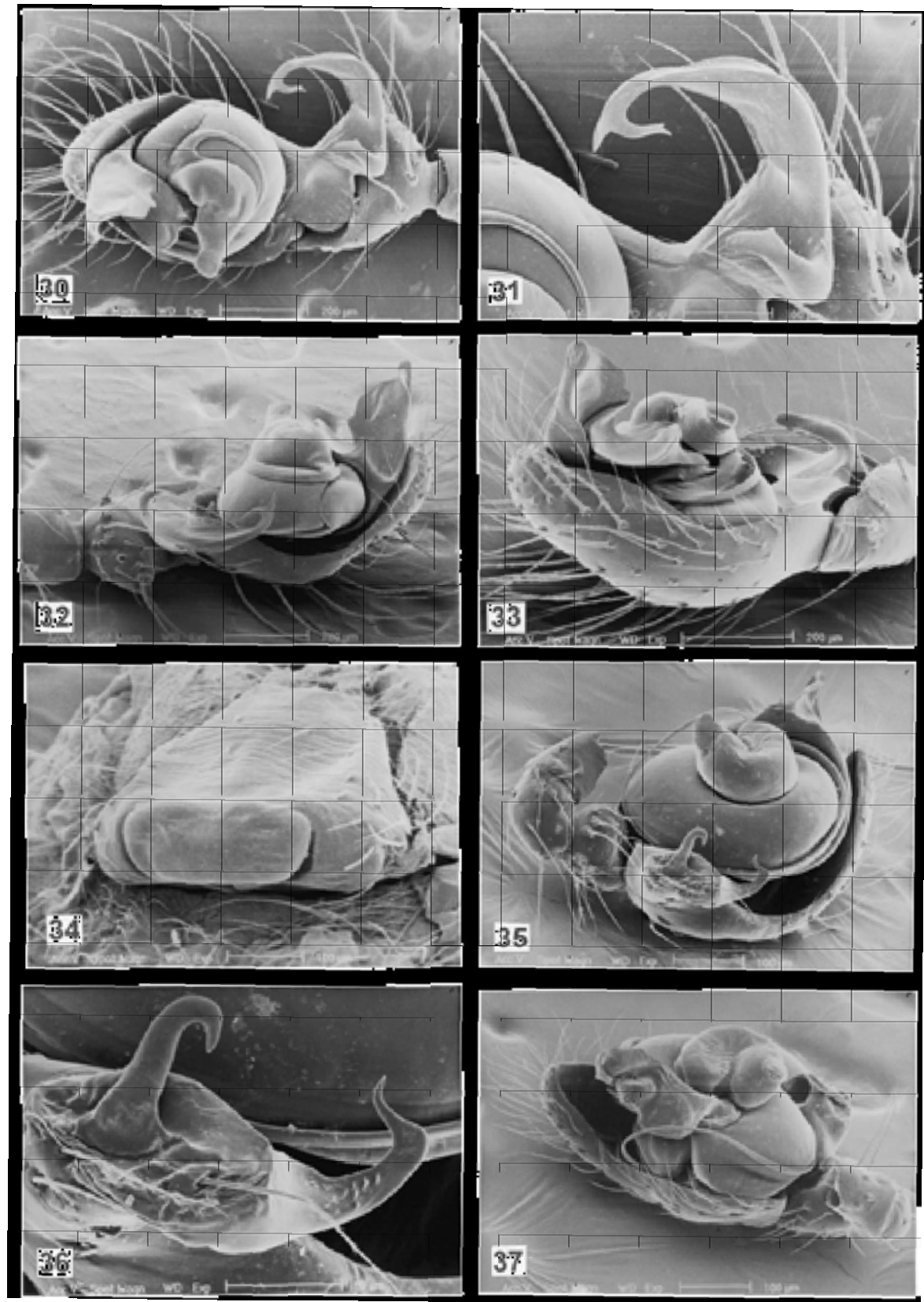

Figs. 30-37. Nesticus potreiro sp. nov.: 30, male palpus, ventral; 31, paracymbium, ventral; 32, male palpus, retrolateral; 33, prolateral; 34, female epigynum, posterior. Nesticus brignolii sp. nov.: 35 , male palpus, retroventral; 36, paracymbium, retroventral; 37, male palpus, proventral. 


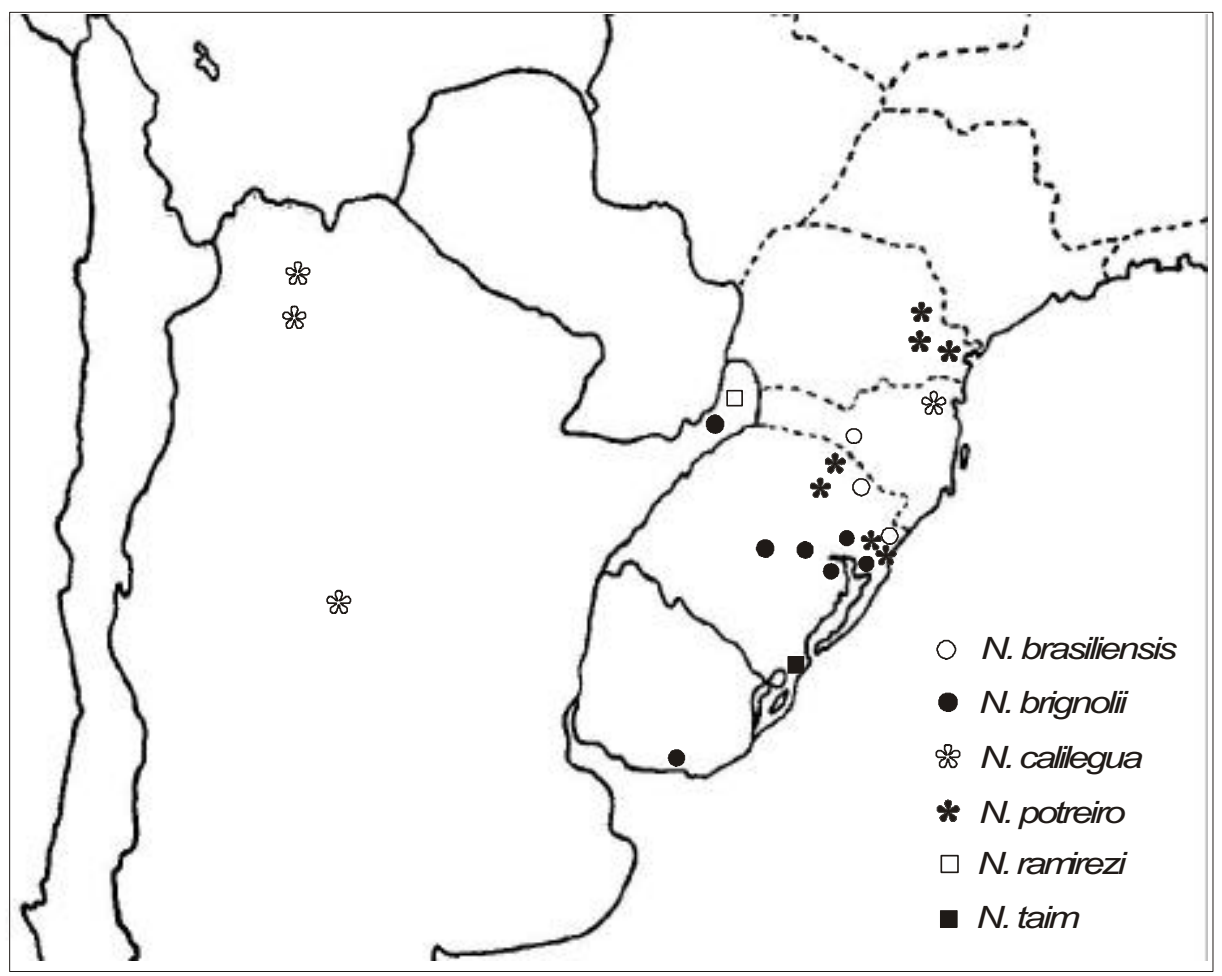

Fig. 38. Geographical distribution of Nesticus species.

Description. Male (holotype). Carapace yellowish with some slightly darkened dorsal lines forming a trident coinciding with bristles disposition; borders marbled with black; conspicuous fovea. Chelicerae yellow. Endites yellow, mesial apex white. Labium yellow, apex white. Sternum yellow, lightly shaded with black, narrow at the distal end. Legs yellow. Abdomen dorsum dark gray with yellowish transversal and median longitudinal spots; laterals and venter colored as dorsum. Epigastric plates white-yellowish.

Measurements. Total length 2.96. Carapace 1.40 long, 1.22 wide, 0.50 high. Clypeus 0.26 high. Eyes diameter and interdistances: AME 0.08, ALE 0.10, PME 0.10, PLE 0.12, AME-AME 0.06, AME-ALE 0.04, PME-PME 0.08, PME-PLE 0.04. MOQ 0.22 dorsal view, 0.18 frontal, 0.20 anterior wide, 0.28 posterior. Chelicerae 0.46 long, 0.26 wide. Endites 0.44 long, 0.24 wide. Labium 0.16 long, 0.28 wide. Sternum 0.90 long, 0.78 wide. Abdomen 1.56 long, 1.26 wide, 0.64 high. Legs: I. femur 2.10, patella 0.54 , tibia 2.02 , metatarsus 1.85 , tarsus 0.86 , total 7.37; II. 1.68, 0.54, 1.45, 1,35, 0.69, 5,71; III. 1.35, 0.44, 1.01, 1.06, 0.61, 4.47; IV. $1.85,0.49,1.65,1.68,0.76,6.43$. Palpus. $0.80,0.24,0.34,-, 0.80,2.18$.

Female paratype (MACN 10033). Carapace yellow, slightly shaded with black, more evident on the laterals. Dorsum with three lines forming a trident, coincident with bristles distribution. Clypeus shaded of black. Endites and labium like in male. Sternum with black pigment. Legs yellow. Abdomen dorsally grayish at back, anteriorly yellowish with gray 
triangular spots. Epigastric plates yellow.

Measurements. Total length 2.80. Carapace 1.20 long, 1,04 wide, 0.40 high. Clypeus 0.26 high. Chelicerae 0.54 long, 0.24 wide. Endites 0.24 long, 0.26 wide. Labium 0.14 long, 0.26 wide. Sternum 0.80 long, 0.72 wide. Eye diameters and interdistances: AME 0.08, ALE 0.10, PME 0.10, PLE 0.10, AME-AME 0.06, AME-ALE 0.04, PME-PME 0.08, PME-PLE 0.04. MOQ 0.20 long, dorsal view, 0.16 , frontal view, 0.16 anterior wide, 0.26 posterior wide. Abdomen 1.70 long, 1.44 wide, 1.46 high. Legs: I. femur 1.90, 0.54, 1.46, 1.42, 0.78, 6.10; II. 1.30, 0.50, 1.02, 1.02, 0.64, 4.48; III. 1.06, 0.44, 0.74, 0.82, 0.54, 3.60; IV. 1.90, 0.50, 1.30, 1.24, $0.70,5.64$. Palpus $0.56,0.20,0.30,-, 0.58,1.64$.

Variation. Total length: 2.52-3.16 males, 2.96-3.80 females. Carapace length 1.20-1.60 males, 1.36-1.72 females.

Natural history. Distribution in very different ecossystems (Subandine to Atlantic Forest). Habitus unknown.

Distribution. Argentina, Salta, Cordoba and Jujuy; Brazil, Santa Catarina (fig. 38).

Acknowledgments. To curators for borrowed material. To Albérico Nogueira de Queiróz for provide contact with the MHNG, allowing us to examine the types of Nesticus brasiliensis. To Erica H. Buckup, Maria Aparecida Marques (MCN), Gustavo Hormiga (George Washington University) and anonymous revisors, for suggestions.

\section{REFERENCES}

Brignoli, P. M. 1979. Ragni del Brasile V. Due nuovi generi e quattro nuove specie dello stato di Santa Catarina (Araneae). Revue suisse Zool., Genève, 86(4):913-924.

Coyle, F. A. \& McGarity, A. C. 1991. Two new species of Nesticus spiders from southern Appalachians (Araneae, Nesticidae). J. Arachnol., New York, 19(3):161-168.

Gertsch, W. J. 1984. The spider family Nesticidae (Araneae) in North America, Central America and West Indies. Austin, Texas Memorial Museum, 91p. (Bulletin, 31)

Lehtinen, P. T. \& SaAristo, M. I. 1980. Spiders of the Oriental-Australian region. II. Nesticidae. Ann. Zool. Fenn., Turku, 17:17-47.

Platnick, N. I. 2002. The world spider catalog, version 3.0. American Museum of Natural History, online at http://research.amnh.org/entomology/spiders/catalog81-87/index.html. 10.05.2002.

Recebido em 28.05.2002; aceito em 30.09.2002. 